\title{
Comment on "Electromagnetic resonances in individual and coupled split-ring resonators” [J. Appl. Phys. 92, 2929 (2002)]
}

\author{
R. Marqués, ${ }^{\text {a) }}$ F. Medina, and R. Rafii-El-Idrissi \\ Departamento de Electrónica y Electromagnetismo, Facultad de Física, Universidad de Sevilla, \\ Av. Reina Mercedes s/n, 41012 Sevilla, Spain
}

(Received 3 December 2002; accepted 28 May 2003)

It is pointed out that the recently reported resonances appearing in split-ring resonators are explained by the recently reported theory about the magnetoelectric coupling (or bianisotropy) in the tensor polarizability of these particles. (C) 2003 American Institute of Physics.

[DOI: 10.1063/1.1592317]

Gay-Balmaz and Martin have presented a detailed and indepth numerical and experimental analysis of the so-called split-ring resonators (SRRs). ${ }^{1}$ These particles were recently proposed by Pendry and co-workers ${ }^{2}$ as suitable elementary bricks to build up artificial media with special electromagnetic properties, such as negative magnetic permeability ${ }^{2}$ and/or negative refractive index ${ }^{3,4}$ when combined with artificial plasma media (conducting wire media). The analysis and experiments reported in Ref. 1 confirm the predictions in Ref. 2 about the behavior of an individual SRR when it is placed in an external time-varying magnetic field. Apart from the confirmation of the predictions of Pendry et al., ${ }^{2}$ some interesting results concerning the behavior of these particles are also reported in Ref. 1. Among these results, it can be found the excitation of a resonance when the SRR is illuminated by an external electromagnetic wave with the magnetic field perpendicular to the particle axis [the so-called "perpendicular" polarization in Fig. 1(a) of Ref. 1]. Since, in this case, the magnetic flux across the rings vanish, this behavior is not expected from the theory reported in Ref. 2 and deserves a comment in order to clarify the underlying physics.

In fact, some months before the publication of Ref. 1, the authors of this comment published a theory about the relevant polarizabilities of the SRR particle ${ }^{5}$ introducing some refinements on previously reported models. This theory-which could not be known by the authors of Ref. 1 at the date of the submission-completes the former model of Ref. 2 by taking into account magnetoelectric couplings in the particle. In brief, it can be said that four resonant polarizabilities are predicted in: $:^{5}$ the so-called magnetic, $\alpha_{z z}^{m m}$, electric, $\alpha_{y y}^{e e}$, and magnetoelectric, $\alpha_{y z}^{e m}=-\alpha_{z y}^{m e}$, polarizabilities (see Ref. 5 for notation and coordinate axis definitions). The first of these polarizabilities is accounted for in the theory reported in Ref. 2 and it is responsible for one of the resonances shown in Fig. 2 of Ref. 1 (the one corresponding to parallel polarization). Although the remaining

${ }^{\text {a)} E l e c t r o n i c ~ m a i l: ~ m a r q u e s @ u s . e s ~}$ polarizabilities are not predicted in Ref. 2, they do lead to physical effects. In particular, the $\alpha_{y y}^{e e}$ polarizability should produce a resonant absorption peak when the particle is placed in an external electric field polarized as in Fig. 1(a) of Ref. 1 (i.e., perpendicular to the particle axis and to the straight line in the plane of the ring passing through the ring slits). The $\alpha_{z y}^{m e}$ and $\alpha_{y z}^{e m}$ polarizabilities also yield a resonant absorption peak for both "parallel" and perpendicular polarizations of the incident wave shown in Fig. 1(a) of Ref. 1.

All these resonances are reported in Ref. 1, Figs. 2-5, as a result of both experiments and computer simulations. It is also of interest the fact that no resonant absorption was observed for incident waves with the perpendicular polarization shown in Fig. 1(b) of Ref. 1. This fact is also consistent with the theory reported in Ref. 5, since no resonant polarizabilities are predicted in such a theory that could be excited by incident waves with this field polarization. The relative strength of the resonances (which are weaker for perpendicular polarization) can be also explained from the numerical values of the different polarizabilities computed with the model in Ref. 5.

Therefore, it can be concluded that the results reported in Ref. 1 provide an experimental and numerical verification of the theory reported in Ref. 5. Although in Ref. 5, indirect numerical and experimental evidence of the theory was given, we think that the experiments and numerical simulations in Ref. 1 provide a much more direct verification of such a theory. The aim of this comment is to point out this fact and to recognize the contribution of the authors of Ref. 1 to this particular problem.

${ }^{1}$ P. Gay-Balmaz and O. J. F. Martin, J. Appl. Phys. 92, 2929 (2002).

${ }^{2}$ J. B. Pendry, A. J. Holden, D. J. Robbins, and W. J. Stewart, IEEE Trans. Microwave Theory Tech. 47, 2075 (1999).

${ }^{3}$ D. R. Smith, W. J. Padilla, D. C. Vier, S. C. Nemat-Nasser, and S. Schultz, Phys. Rev. Lett. 84, 4184 (2000).

${ }^{4}$ R. A. Shelby, D. R. Smith, and S. Schultz, Science 292, 77 (2001).

${ }^{5}$ R. Marqués, F. Medina, and R. Rafii-El-Idrissi, Phys. Rev. B 65, 144440 (2002). 